\title{
Self-Nanoemulsifying Drug Delivery System of Tetrandrine for Improved Bioavailability: Physicochemical Characterization and Pharmacokinetic Study
}

\author{
Chunxia Liu, ${ }^{1,2}$ Li Lv, ${ }^{2}$ Wei Guo, ${ }^{3}$ Lan Mo, ${ }^{3}$ Yaoxing Huang, \\ Guocheng $\mathrm{Li}\left(\mathbb{D},{ }^{1,2}\right.$ and Xingzhen Huang $\mathbb{D}^{3}$ \\ ${ }^{1}$ Department of Pharmacy, Zengcheng District People's Hospital of Guangzhou, Guangzhou 511300, Guangdong, China \\ ${ }^{2}$ Department of Pharmacy, Sun Yat-Sen Memorial Hospital, Sun Yat-Sen University, Guangzhou 510120, Guangdong, China \\ ${ }^{3}$ School of Pharmacy, Guangxi Medical University, Nanning 530021, Guangxi, China \\ Correspondence should be addressed to Guocheng Li; guochenglisyx@126.com \\ and Xingzhen Huang; huangxingzhen@gxmu.edu.cn
}

Received 10 May 2018; Accepted 27 August 2018; Published 27 September 2018

Academic Editor: Sanyog Jain

Copyright (C) 2018 Chunxia Liu et al. This is an open access article distributed under the Creative Commons Attribution License, which permits unrestricted use, distribution, and reproduction in any medium, provided the original work is properly cited.

\begin{abstract}
The main purpose of this study was to investigate the potential of self-nanoemulsified drug delivery system (SNEDDS) to improve the oral bioavailability of tetrandrine (Tet). SNEDDS was developed by using rational blends of excipients with good solubilizing ability for Tet which was selected based on solubility studies. Further ternary phase diagram was constructed to determine the selfemulsifying region. The optimal formulation with the best self-nanoemulsified and solubilization ability consisted of $40 \%$ (w/w) oleic acid as oil, $15 \%(\mathrm{w} / \mathrm{w})$ SPC and 30\% (w/w) Cremophor RH-40 as surfactant, and 15\% (w/w) PEG 400 as cosurfactant. The average droplet size and zeta-potential of the optimal Tet SNEDDS were $19.75 \pm 0.37 \mathrm{~nm}$ and $1.87 \pm 0.26 \mathrm{mv}$, respectively. The dissolute rate of Tet SNEDDS in various dissolution media was remarkably faster than Tet commercial tablet. Moreover, in vivo pharmacokinetic study results show that significant increase $(\mathrm{p} \leq 0.05)$ in the peak concentration (Cmax) and the area under the curve (AUC) of Tet was observed after the oral administration of Tet SNEDDS and the absorption of Tet from SNEDDS resulted in approximately 2.33-fold increase in oral bioavailability compared with the commercial tablet. Our research suggests that the prepared Tet SNEDDS could be a good candidate for improved the dissolution and oral bioavailability of Tet.
\end{abstract}

\section{Introduction}

Tetrandrine (Tet) is a bisbenzyltetrahydroisoquinoline alkaloid (Figure 1), which is found in the Chinese medicinal herb, han-fang-chi (or fen-fang-qi, Stephania tetrandra S.Moore) [1]. The first pharmacological and toxicological study of Tet was published in 1937 and demonstrated its hypotensive and cardiodepressant effects [2]. According to the literature, for over half a century, Tet has attracted large amount of interest because it shows a variety of pharmacological effects, including antiarrhythmia, antiatherogenic, antisilicosis, antihypertension, and immunomodulation effects [3-8]. Tet has been mainly used for early mild hypertension in clinical therapeutics and for severe hypertensive and hypertensive crises. The antihypertensive effect is made through a calcium antagonist mechanism $[9,10]$. In recent years, Tet has also attracted much attention due to its obvious effect on experimental silicosis [11-15]. There is a growing body of evidence that Tet exerts anticancer effects against a variety of cancers, including leukemia, hepatocellular carcinoma, gastric cancer, colon cancer, lung cancer, glioma, nasopharyngeal carcinoma, prostate cancer, breast cancer, and bladder cancer [16-21].

Although Tet has potentially considerable value in clinic, some problems have arisen that have limited its clinical application. In general, a large dose (6 to 15 tablets per day) is required for the current commercially available tablets [22]. Additionally, Tet's poor solubility in physiological environment (Tet saturation $=0.015 \mathrm{mg} / \mathrm{mL}$ in $\mathrm{pH} 7.4$ phosphate buffered saline (PBS)) caused by the presence of a quaternary 


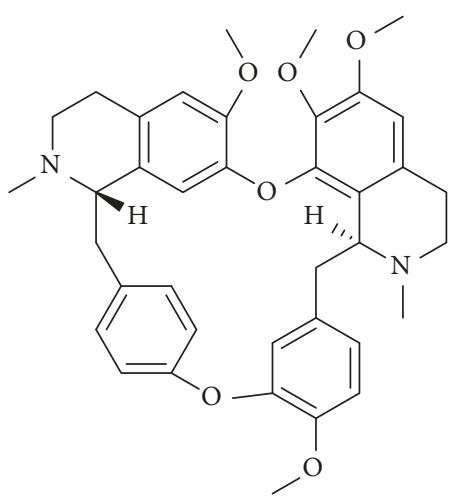

FIgure 1: Molecular structure of tetrandrine (Tet).

ammonium cation [23] contributes to its low and variable oral bioavailability, gastric intestine and kidney damage, and bad patient compliance [24]. In recent years, many pharmaceutical methods have been investigated to improve the bioavailability of Tet, such as lipid nanocapsules, nanoparticles, ethosomes, and microspheres [25]. However, most of tetrandrine based nanoparticles were used for injection and seldom used in oral administration.

Investigation of novel drug delivery systems to enhance the solubility and improve bioavailability of Tet is considered a matter of urgency [26]. In recent years, various strategies have been considered to overcome the problems associated with oral absorption and bioavailability such as microsphere, microcapsule, nanoemulsified drug delivery system (SNEDDS), and nanoparticles [23]. A high speed disperser and a high pressure emulsifier, generating particles with an average diameter of about $200 \mathrm{~nm}$ in vitro has shown some success for Tet encapsulation [27] and coencapsulated doxorubicin and bromotetrandrine lipid nanoemulsions have shown some potential of treatment in breast cancer animal models [28].

SNEDDS is an isotropic mixture of oils, surfactant, hydrophilic cosurfactant, and drug substances, which form a fine oil-in-water microemulsion when introduced into aqueous media while being gently agitated by the digestive motility of the stomach and intestine. SNEDDS is a relatively recent term used to indicate formulations with a globule size less than $100 \mathrm{~nm}$ [29]. It has excellent efficiency in increasing the dissolution rate, promoting oral absorption and increasing the bioavailability of poorly water-soluble drugs [30, 31], and has shown a lot of success for herbal drugs [32]. There are some commercially successful formulations such as Neoral $^{\circledR}$ (cyclosporine A), Fortovase ${ }^{\circledR}$ (saquinavir), Agenerase $^{\circledR}$ (amprenavir), and Norvir ${ }^{\circledR}$ (ritonavir) in clinical use.

Extensive review of literature reveals lack of reports about the bioavailability improvement of poorly water-soluble Tet using SNEDDS. Thus, the objective of the present study was to formulate Tet SNEDDS to improve Tet's oral bioavailability. To achieve this, Tet's solubility in various excipients was evaluated and that with highest solubility of Tet were selected oil, surfactant, and cosurfactant of Tet SNEDDS. The prepared Tet SNEDDS was evaluated for morphological, droplet size, zeta-potential, thermodynamic stability, and in vitro dissolution study. Furthermore, an in vivo pharmacokinetic study was performed to study Tet's pharmacokinetic behavior and bioavailability enhancement after oral administration.

\section{Materials and Methods}

2.1. Materials. Tet (purity $>98.0 \%$ ) was obtained as a gift sample from Beihai Sunshine Pharmaceutical co., Ltd. (Beihai, China). A Tet tablet (containing $20 \mathrm{mg}$ Tet in one tablet with excipients including starch, dextrin, and magnesium stearate) was manufactured by Beihai Sunshine Pharmaceutical co., Ltd (Beihai, China). Oleic acid, Tween 60, and Tween 80 were purchased from Tianjin Damao Chemical Reagent Factory (Tianjin, China). Soybean oil, isopropyl acetate, olive oil, ethyl linoleate, cremophor RH-40, and isopropyl myristate (IPM) were purchased from Chengdu Gracia Chemical Technology co., Ltd (Chengdu, China). Ethyl oleate, butyl oleate, and soybean phosphatidylcholine (SPC) were purchased from Tianjin Xing Fu Fine Chemical Industry Research Institute (Tianjin, China). Isopropanol, glycerol, absolute alcohol, and $\mathrm{PEG}_{400}$ were purchased from Chengdu Kelon Chemical Reagent Factory (Chengdu, China). Methanol and acetonitrile (chromatographic grade) were from Thermo Fisher Scientific (China) Co., Ltd. All other chemicals used were of analytic grade.

2.2. Determination of Tet Solubility. The solubility of Tet was determined in selected oils, surfactants, and cosurfactants by pouring an excess of Tet into $1 \mathrm{~mL}$ of each excipient. The obtained mixtures were mixed using a vortex mixer. And then the mixture was kept in a water bath at $37 \pm 0.5^{\circ} \mathrm{C}$ for 72 hours. After equilibrium was achieved, the equilibrated samples were centrifuged at $3000 \mathrm{rpm}$ for $15 \mathrm{~min}$ and excess insoluble Tet was discarded by filtration using a $0.45 \mu \mathrm{m}$ membrane filter. The concentration of Tet was determined by a high performance liquid chromatography (HPLC) method described below. The experiments were performed in triplicate.

2.3. HPLC Analysis. The amount of Tet in various excipients was quantified by a validated HPLC method [33], equipped with an ultraviolet detector (Agilent 1260 Liquid Chromatography, American). Tet was separated on a $\mathrm{RP}-\mathrm{C}_{18}$ column (ODS $\mathrm{C}_{18}, 200 \mathrm{~mm} \times 4.6 \mathrm{~mm}, 5 \mu \mathrm{m}$ ) using methanolacetonitrile-water $(3: 1: 1, \mathrm{v}: \mathrm{v}: \mathrm{v})$ plus $0.06 \%$ diethylamine as mobile phase, at a flow rate of $1.0 \mathrm{~mL} / \mathrm{min}$ with the detection wavelength at $282 \mathrm{~nm}$. The column temperature was maintained at $25^{\circ} \mathrm{C}$.

2.4. Preparation of SNEDDS. For preparation of Tet SNEDDS, the content of Tet was fixed at a constant level $(1.0 \% \mathrm{w} / \mathrm{w}$ of excipients). Firstly, Tet was dissolved by cosurfactant, and then the mixture containing the calculated amount of oil and surfactant was mixed by gentle stirring for $10 \mathrm{~min}$ and vortex mixing until the microemulsion formulation was obtained. 
2.5. Pseudo-Ternary Phase Diagram Study. The pseudoternary phase diagrams were constructed with oil, surfactant, and cosurfactant using the water titration method at room temperature: each of them representing an apex of the triangle and the total of them was kept at $100 \%$. The mixture oil and surfactant/cosurfactant at certain weight ratio were titrated with water and mixed by magnetic stirring until equilibrium was reached. The mixtures which were clear or slightly bluish appearance were determined as microemulsions. In the SNEDDS, oleic acid was used as the oil, a mixture of SPC and cremophor RH-40 was used as surfactant and $\mathrm{PEG}_{400}$ was used as cosurfactant. The ratios of SPC to cremophor RH40 by weights $1: 1,2: 1$, and $1: 2$ were studied by constructing a ternary phase diagram. The key factor for SNEDDS is the ratio of surfactant to cosurfactant. Phase diagrams at specific ratios of surfactant to cosurfactant by weights $1: 1,3: 2,2: 1$, and $3: 1$ were taken. Mixtures of surfactant/cosurfactant (at a specific ratio) with oil were prepared at ratios $9: 1,8: 2,7: 3$, $6: 4,5: 5,4: 6,3: 7,2: 8$, and $1: 9$ by weight. Each mixture was titrated with water and visually observed for phase clarity. The microemulsion regions in the diagrams were plotted. Also the microemulsion formulations were selected at desired components ratios.

\subsection{Characterization of SNEDDS}

2.6.1. Morphological Characterization. The morphology of SNEDDS was observed by transmission electron microscopy (Hitachi transmission electron microscope H7650, Japan). SNEDDS was diluted with distilled water and mixed by slightly shaking. One drop of diluted samples was deposited on a film-coated 200 mesh copper specimen grid and allowed to stand for $20 \mathrm{~min}$, after which any excess fluid was removed with the filter paper. The grid was later stained with one drop of $2 \%$ phosphotungstic acid and dried for $15 \mathrm{~min}$ before examination with the transmission electron microscope.

2.6.2. Determination of Droplet Size and Zeta-Potential. $1 \mathrm{~mL}$ SNEDDS was diluted with $50 \mathrm{~mL}$ distilled water in a glass flask and gently stirred. The droplet size distribution and zetapotential of the resultant microemulsions were determined by dynamic light scattering with particle size apparatus (Malvern Zetasizer Nano ZS90, UK). Each sample was analyzed in triplicate.

2.6.3. Thermodynamic Stability Study. The objective of thermodynamic stability is to evaluate the phase separation and effect of temperature variation on SNEDDS formulation. The stability was evaluated by centrifugation, a heating-cooling cycle and a freeze-thaw cycle. Briefly, Tet was diluted with aqueous medium, followed by centrifugation at $15000 \mathrm{rpm}$ for $15 \mathrm{~min}$, and then the mixture was observed visually for phase separation; after SNEDDS formulations were diluted with deionized water $(1: 50)$ and subjected to freeze-thaw cycles $\left(-20^{\circ} \mathrm{C}\right.$ for 2 days) followed by heated to $40^{\circ} \mathrm{C}$ for 2 days, the phase separation and appearance were observed.

2.6.4. In Vitro Dissolution Study. The in vitro dissolution behaviors of Tet from commercial tablet of Tet and Tet
SNEDDS were assessed using the Chinese pharmacopoeia (2010 version) paddle method. The SNEDDS containing Tet $(1.0 \% \mathrm{w} / \mathrm{w}$ of the excipients) were added into hydroxy-propyl methyl cellulose (HPMC) capsules "size 0." A Tet SNEDDS hard capsule/tablet was put into a sinker. Thus, the sinker was loaded with $900 \mathrm{~mL}$ of simulated gastric fluid without enzymes ( $\mathrm{pH}$ 1.2), phosphate buffer $\mathrm{pH} 6.8$, and distilled water (containing $0.5 \%$ of sodium dodecyl sulfate to increase dissolution) at $37 \pm 0.5^{\circ} \mathrm{C}$ with a paddle speed of $100 \mathrm{rpm}$ using a dissolution tester (ZRS-8G, Tianjin University Electronics Co. Ltd, Tianjin, China). $5.0 \mathrm{~mL}$ sample was withdrawn at 5 , $10,20,30,45,60,90,120$, and 180 min and replaced with fresh dissolution medium to keep the volume constant. The release of Tet from the SNEDDS formulation was compared with commercial tablets containing the same quantity of drug. The concentration of Tet in the released sample was determined by the HPLC method described in the above section " 2.3 HPLC analysis."

2.7. Pharmacokinetic Study. The pharmacokinetic experiment was designed to compare the Tet SNEDDS formulation and the Tet commercial tablet. The male Wistar Albino rats (180-220 g) were purchased from Guangxi Medical University Laboratory Animal Centre, China. They were allocated to two groups at random: the first group received the Tet commercial tablet, which was prepared by Tet suspension $(0.25 \% \mathrm{w} / \mathrm{v}$ carboxymethyl cellulose sodium (CMC-Na)) according to the following method: ten tablets were ground to a fine powder with a mortar. The powder was weighed and mixed with $0.25 \%$ CMC-Na solution to make a suspension. The suspension was made and used when needed. The second group received the Tet SNEDDS formulation. Each group consisted of six rats. Tet SNEDDS formulation was given orally or gavage at the same dose of $10 \mathrm{mg} / \mathrm{kg}$ body weight. The dose was based on the median of the clinical dose of Tet tablets, which is between 80 and $120 \mathrm{mg} /$ day/person. Based on an average weight of $60 \mathrm{~kg}$ per person, the dose will be $1.67 \mathrm{mg} / \mathrm{kg} / \mathrm{day}$. Thus, for the rat model the dose was $10 \mathrm{mg} / \mathrm{kg} /$ day. All rats were dosed following an overnight fast. Food was supplied to the rats after $4 \mathrm{~h}$ of the dose administration. In vivo study was approved by the Animal Ethics Committee of Guangxi Medical University.

Blood samples (approximately $0.3 \mathrm{~mL}$ ) were collected from the retroorbital plexus with a heparinized tube at predose and $0.25,0.5,1,2,3,4,6,8,12,24,48$, and $72 \mathrm{~h}$ postdose. Blood samples were centrifuged at $6000 \mathrm{rpm}$ for $10 \mathrm{~min}$. $5 \mu \mathrm{L}$ demethyltetrandrine (internal standard, IS) solution $(10 \mu / \mathrm{mL}), 0.1 \mathrm{~mL}$ acetonitrile and $0.4 \mathrm{~mL}$ ether were added to $0.1 \mathrm{~mL}$ upper plasma. After vortex mixing for $3 \mathrm{~min}$ and centrifugation at $3000 \mathrm{rpm}$ for $15 \mathrm{~min}$, the upper organic layer was transferred and the plasma was extracted by $0.4 \mathrm{~mL}$ ether again. The combined organic phase was evaporated to dryness at $40^{\circ} \mathrm{C}$. Then the residue was dissolved in $100 \mu \mathrm{L}$ methanol and centrifuged at $10000 \mathrm{rmp}$ for $10 \mathrm{~min}$ [34]. After centrifugation, $2 \mu \mathrm{L}$ of clear supernatant was injected into UPLC-MS-MS system for analysis.

The concentration of Tet was determined by UPLC-MSMS according to the following: HPLC analysis was performed 
TABLE 1: Result of solubility studies with Tet in various excipients $(n=3)$.

\begin{tabular}{lccc}
\hline Sample & Excipient & Function in SNEDDS & Solubility $(\mathrm{mg} / \mathrm{mL})$ \\
\hline 1 & Oleic acid & Oil & $8.674 \pm 0.056$ \\
2 & Soybean oil & Oil & $2.372 \pm 0.091$ \\
3 & Isopropyl acetate & Oil & $2.291 \pm 0.117$ \\
4 & Olive oil & Oil & $2.124 \pm 0.065$ \\
5 & Ethyl linoleate & Oil & $3.326 \pm 0.177$ \\
6 & Isopropyl myristate (IPM) & Oil & $4.348 \pm 0.198$ \\
7 & Ethyl oleate & Oil & $1.185 \pm 0.066$ \\
8 & Butyl oleate & Oil & $1.194 \pm 0.064$ \\
9 & Tween-60 & Surfactant & $4.216 \pm 0.208$ \\
10 & Tween-80 & Surfactant & $6.793 \pm 0.324$ \\
11 & SPC & Surfactant & $10.238 \pm 0.199$ \\
12 & Cremophor RH- 40 & Surfactant & $12.645 \pm 0.252$ \\
13 & Isopropanol & Co-surfactant & $2.564 \pm 0.088$ \\
14 & Glycerol & Co-surfactant & $3.455 \pm 0.100$ \\
15 & PEG400 & Co-surfactant & $9.584 \pm 0.225$ \\
16 & Absolute alcohol & Co-surfactant & $8.449 \pm 0.165$ \\
\hline
\end{tabular}

using the Dionex UltiMate 3000 with a binary pump, an online degasser, an auto-sampler, and a column temperature controller. Chromatographic separations were performed on a Hypersil GOLD $\mathrm{C}_{18}$ column $(100 \mathrm{~mm} \times 2.1 \mathrm{~mm}, 1.9 \mu \mathrm{m})$ protected by a $\mathrm{C}_{18}$ guard column $(10 \mathrm{~mm} \times 2.1 \mathrm{~mm}, 5 \mu \mathrm{m})$ at $35^{\circ} \mathrm{C}$. The mobile phase consisted of methanol-water $(50: 50$, $\mathrm{v} / \mathrm{v})$. The flow rate was set at $0.2 \mathrm{~mL} / \mathrm{min}$.

MS analysis was carried out on a Thermo Scientific TSQ Quantum Access MAX triple stage quadrupole mass spectrometer with an electrospray ionization (ESI) source running in a positive-ionization mode. The typical ion source parameters were spray voltage: $3500 \mathrm{~V}$; sheath gas pressure $\left(\mathrm{N}_{2}\right)$ : 30 units; auxiliary gas pressure $\left(\mathrm{N}_{2}\right)$ : 10 units; ion transfer tube temperature: $400^{\circ} \mathrm{C}$; collision gas (Ar): $1.5 \mathrm{mTorr}$; Q1/Q3 peak resolution: 0.7 Da; scan width: 0.002 Da; samples were analyzed via selective-reaction monitoring (SRM) with monitoring ion pairs at $\mathrm{m} / \mathrm{z} 623 \longrightarrow 381$ for Tet, $\mathrm{m} / \mathrm{z} 609$ $\longrightarrow 367$ for IS. The scan dwell time was set at 0.1 s for every channel. All data collected in centroid mode were acquired and processed using Xcalibur 2.2 software (Thermo Fisher Scientific Inc., USA). Peak area ratio of Tet to IS was calculated, and the calibration curve was established with the ration as Y-axis while the corresponding nominal concentrations of Tet as $\mathrm{X}$-axis.

2.8. Data Analysis. Statistical evaluation was performed by Student t-test of paired observations to analyze different concentrations of Tet. The pharmacokinetic data were processed by noncompartmental analysis using the DAS 2.0 software package (Chinese Pharmacological Society).

\section{Results}

3.1. Solubility Studies of Tet in Various Excipients. The objective of solubility studies is to identify the most suitable oil, surfactant, and cosurfactant which have a good solubilizing capacity for Tet. The concentration of Tet in various excipients at $37^{\circ} \mathrm{C}$ was determined by HPLC, and solubility results are presented in Table 1. If excipients with high drug solubility are chosen to prepare SNEDDS formulation, then the amount of formulation to be administered will be small. It is clear from the table that the highest solubility of Tet is in oleic acid as the oil and in the surfactants, SPC, and cremophor RH-40, with the cosurfactant, $\mathrm{PEG}_{400}$, and absolute alcohol. However, absolute alcohol was not selected as cosurfactant due to its volatility that would result in precipitation or crystallization of drugs. So in the preparation of SNEDDS, oleic acid was used as oil, SPC and cremophor $\mathrm{RH}-40$ were used as surfactant, and $\mathrm{PEG}_{400}$ was used as cosurfactant.

3.2. Construction of Pseudo-Ternary Phase Diagrams. Pseudo-ternary phase diagrams were constructed to identify the microemulsion regions for optimizing the concentrations of oil, surfactant, cosurfactant, and their mixing ratios. The visual test measured the apparent spontaneous of emulsion formation. Screening of the mixed surfactant was based on the pseudo-ternary phase diagram. As shown in Table 1, for surfactant, Tet had the highest solubility in cremophor $\mathrm{RH}-40$, followed by SPC. The maximum region of selfmicroemulsion was obtained when mixture of SPC and cremophor RH-40 in the ratio of 1:2 was used. Therefore, the optimal of SPC to cremophor RH-40 was selected to be $1: 2$. The phase diagrams containing oleic acid, SPC and cremophor RH-40, and $\mathrm{PEG}_{400}$ were shown in Figure 2. As shown in Figure 2, microemulsion formation area was increased with an increase in 3:1 (the ratio of surfactant to cosurfactant) and 3:2 (the ratio of mixtures including surfactant and cosurfactant to oil). There will be a loss of flow if the surfactant to cosurfactant ratio is more than 3:1. According to these results, the following SNEDDS formulation was established: $40 \%(\mathrm{w} / \mathrm{w})$ oleic acid as oil, $15 \%$ (w/w) SPC and 30\% (w/w) Cremophor RH-40 as surfactant, and $15 \%(\mathrm{w} / \mathrm{w}) \mathrm{PEG}_{400}$ as cosurfactant. 


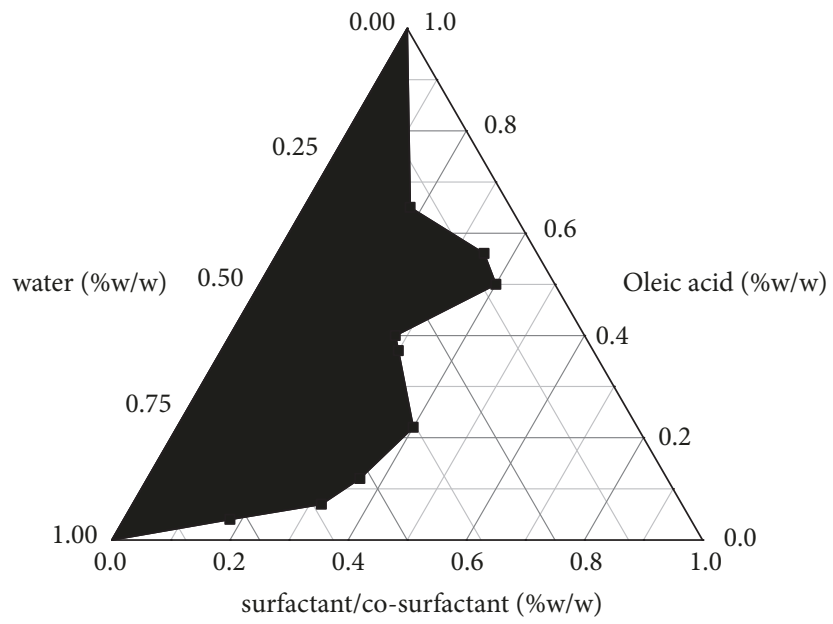

(a)

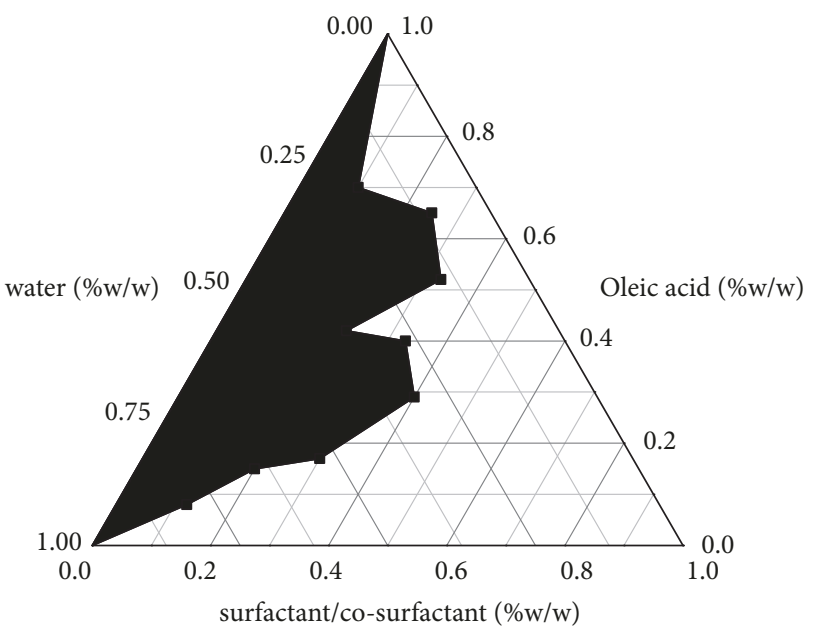

(c)

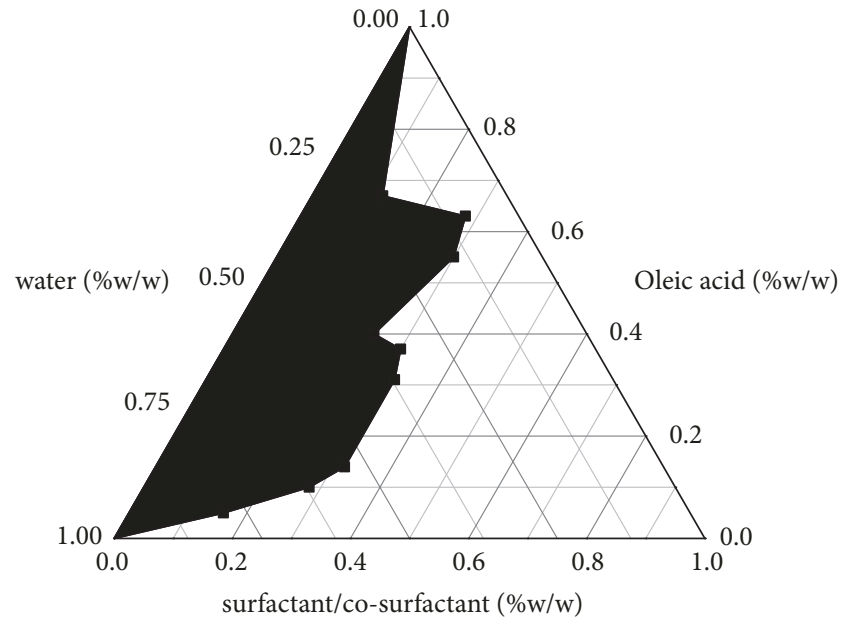

(b)

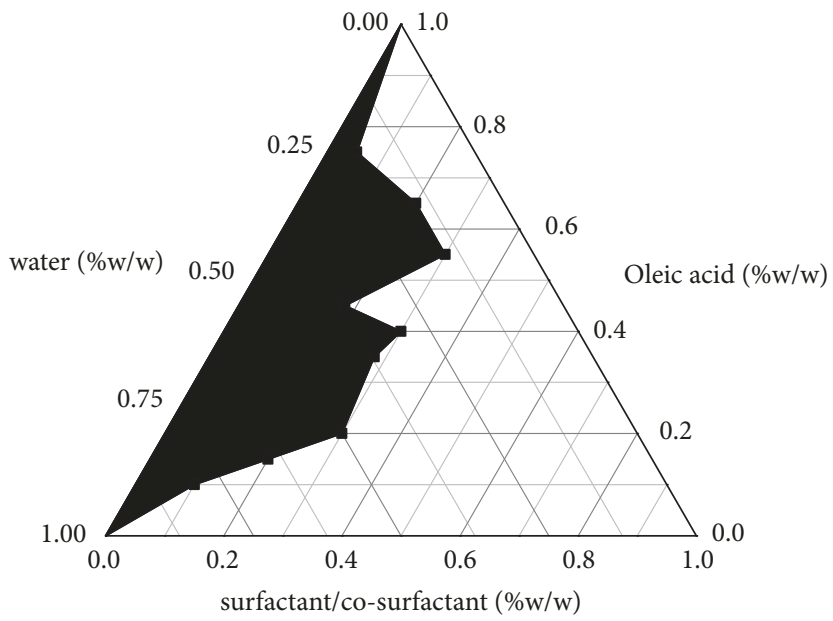

(d)

FIGURE 2: Pseudo-ternary phase diagram consisting of oleic acid, the mixture of surfactant (SPC/cremophor RH-40, 1:2), and PEG 400 with surfactant/cosurfactant ratios of (a) $1: 1$, (b) $3: 2$, (c) $2: 1$, and (d) $3: 1$. The blank region represents oil/water microemulsion formation area.

\subsection{Characterization of SNEDDS}

3.3.1. Morphological Characterization. In order to observe the oily droplets, the SNEDDS of Tet was turned into microemulsion by diluting with distilled water. A representative transmission electron microscope picture is shown in Figure 3. From the figure it is evident that all the oil droplets were of spherical in shape.

3.3.2. Droplet Size and Zeta-Potential Analysis. As shown in Figure 4, the mean droplet sizes of diluted SNEDDS preconcentrates were very low, and all were found to be in the nanometric range $(<100 \mathrm{~nm})$. The average droplet size of Tet microemulsion was $19.75 \pm 0.37 \mathrm{~nm}$ and showed Gaussian distribution. The SNEDDS of Tet was diluted with distilled water. The resultant zeta-potential was $1.87 \pm 0.26 \mathrm{mv}$, which indicated that the microemulsion droplets had no charge (see Figure 5).

3.3.3. Thermodynamic Stability Studies. For lipid-based dosage forms, stability is crucial to their performances which

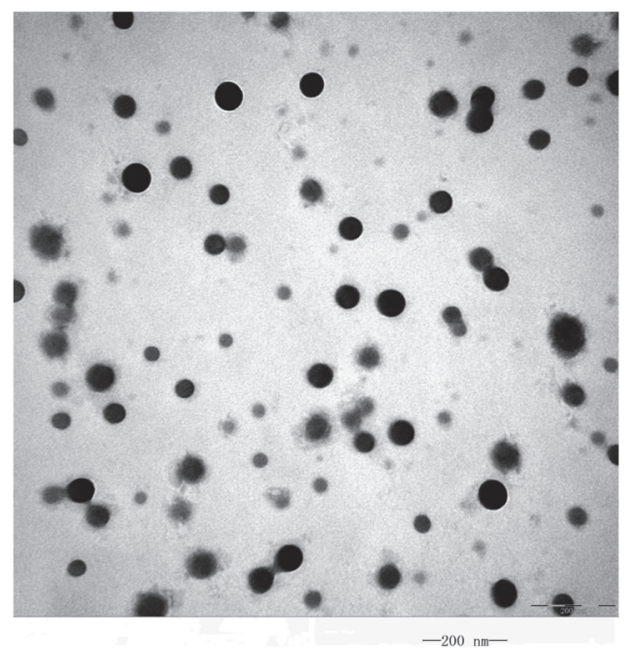

Figure 3: Transmission electron microscopy photo of Tet microemulsion $(\times 100000)$. 


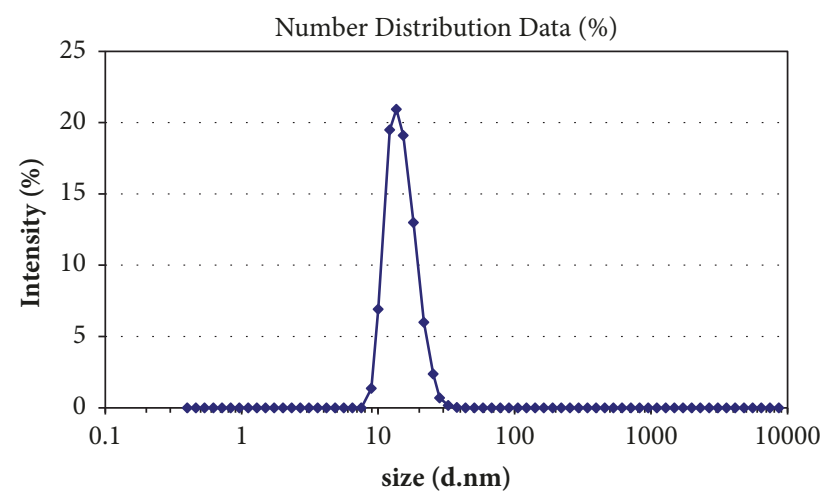

(a)

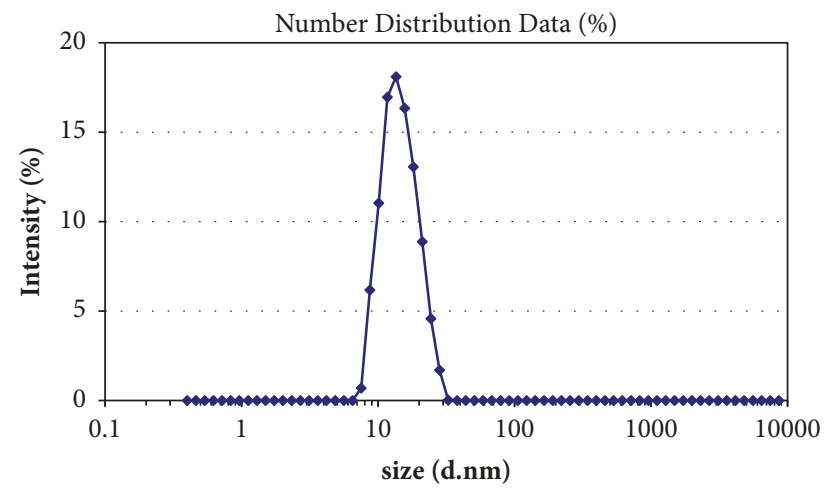

(b)

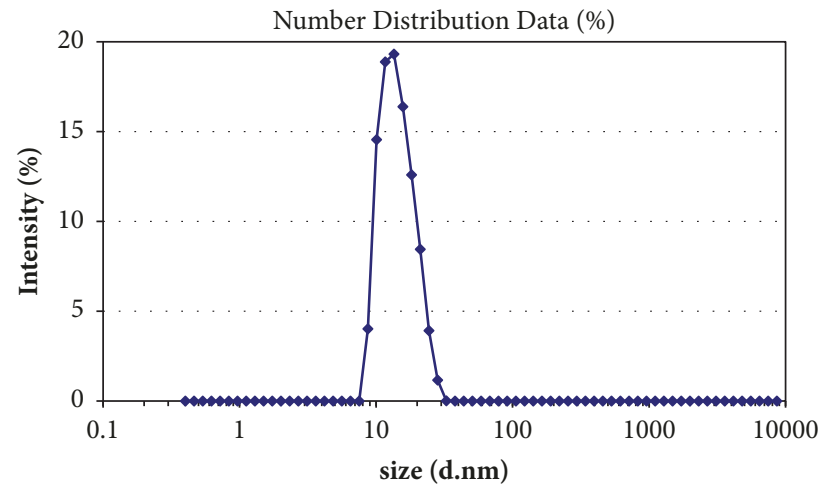

(c)

FIgURE 4: $(\mathrm{a}-\mathrm{c})$ Droplet size distribution by intensity $(\mathrm{n}=3)$.

can make an adverse effect in the form of precipitation of the drug in the excipient matrix. In thermodynamic stability studies, formulation selected was subjected to stress test like centrifugation (at $15000 \mathrm{rpm}$ for $15 \mathrm{~min}$ ) and freeze-thaw test $\left(-20^{\circ} \mathrm{C}\right.$ for 2 days followed by $+40^{\circ} \mathrm{C}$ for 2 days). The SNEDDS formulation was found to be stable under these stressed conditions. Since the Tet SNEDDS formulation was stable, a metastable formation is thus avoided and frequent tests need not be performed during storage.

3.3.4. In Vitro Dissolution Study. An in vitro dissolution profile of the optimized Tet SNEDDS formulation was performed to compare with the commercial tablet, and the dissolution of drug from various media was evaluated. The

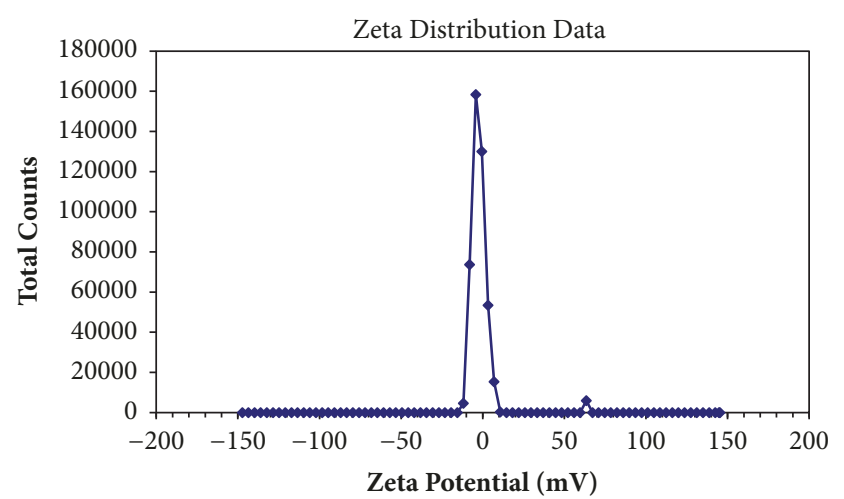

(a)

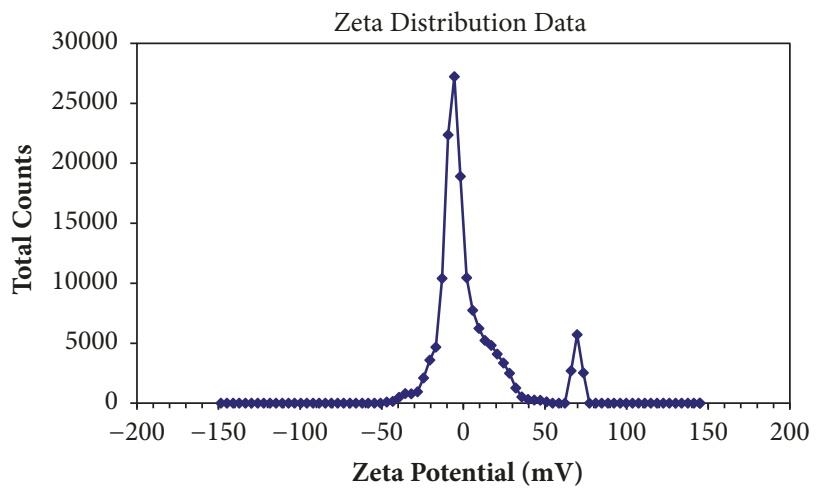

(b)

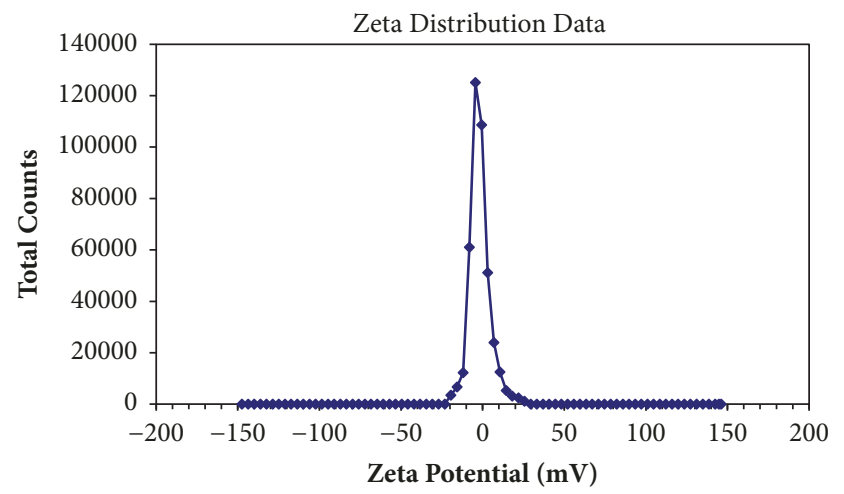

(c)

FIGURE 5: $(\mathrm{a}-\mathrm{c})$ zeta- potential distribution $(\mathrm{n}=3)$.

dissolution of Tet from SNEDDS and Tet commercial tablet formulation in various medium were presented in Figure 6 and showed that the Tet SNEDDS formulation allowed 85\% dissolution of Tet within 20 min whereas the commercial tablet showed less than $80 \%$ of Tet within $180 \mathrm{~min}$. The dissolute rate of the Tet SNEDDS formulation in various dissolution media was remarkably faster than commercial tablet due to the small droplet size. In order to establish the kinetics of drug dissolution, various mathematical models including zero-order, first-order, Higuchi, Korsmyer-Peppas, Hixson-Crowell, and Weibull were used. The assessment of the models is presented in Table 2 that provides the model selection criteria (MSC), by selecting the model with the highest MSC, it was found that the drug dissolution from 


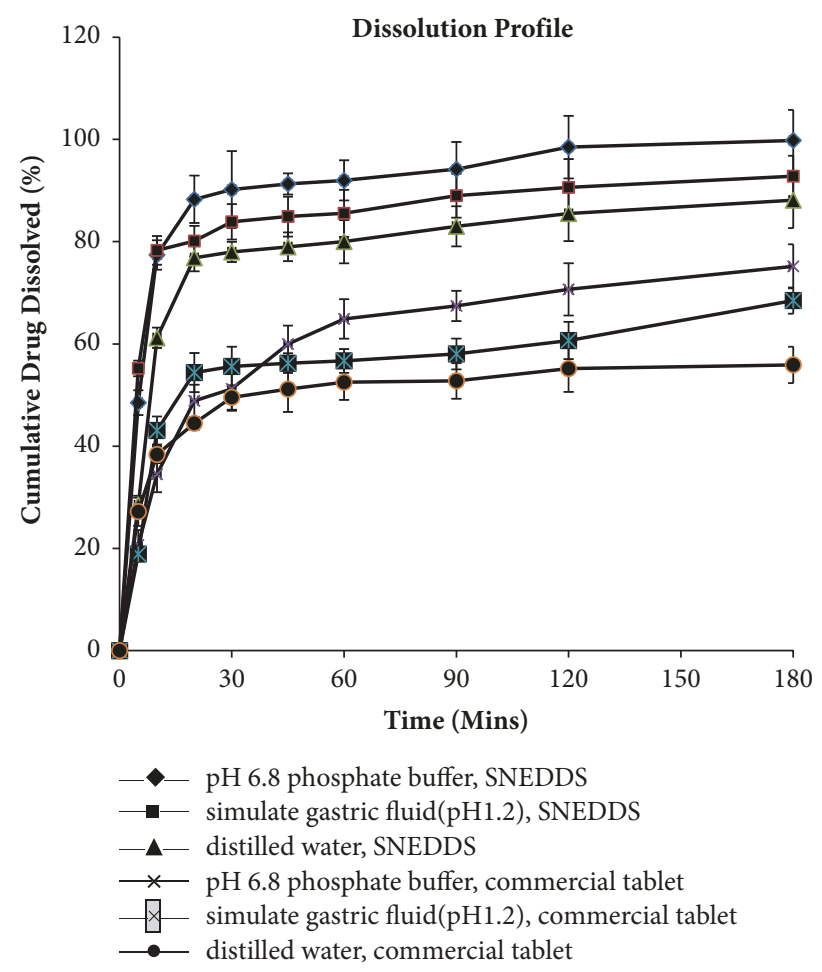

FIgURE 6: In vitro profiles of Tet from SNEDDS and Tet commercial tablet formulation in various medium at $37^{\circ} \mathrm{C}$ with a paddle speed of $100 \mathrm{rpm}$ using a dissolution tester (ZRS-8G, Tianjin University Electronics Co. Ltd, Tianjin, China). Each value represents the mean $\pm \operatorname{SD}(n=6)$.

TABLE 2: The model selection criteria of various mathematical models for the drug dissolution from SNEDDS.

\begin{tabular}{lc}
\hline Mathematical models & Model selection criteria (MSC) \\
\hline Zero-order & -1.3324 \\
First-order & 1.6415 \\
Higuchi & -0.2989 \\
Korsmyer-Peppas & 3.0797 \\
Hixson-Crowell & -0.2690 \\
Weibull & 5.7438 \\
\hline
\end{tabular}

SNEDDS best fitted with Weibull Distribution Mode: $y=$ $5.67 \times 10^{6} \times\left(1-e\left(-(x-4.99)^{0.05} /\left(7.99 \times 10^{4}\right)\right)\right), \mathrm{r}^{2}=0.9996$.

3.4. Pharmacokinetic Study. Figure 7 presents the plasma concentration vs time curve for Tet SNEDDS formulation and Tet commercial tablet after a single dosage of oral administration in male rats. At all the indicated time points, Tet blood concentrations in rats treated with Tet SNEDDS were significantly higher than those treated with Tet commercial tablet. Pharmacokinetic parameters are shown in Table 3. The results indicated that the average of $\mathrm{T}_{\max }$ of the SNEDDS formulation and commercial tablet was $3.8 \mathrm{~h}$ and $6.6 \mathrm{~h}$, respectively, which meant that the Tet SNEDDS formulation showed a faster rate of absorption than the Tet commercial tablet. The peak plasma concentration $\left(\mathrm{C}_{\max }\right)$ and the area under the curve $\left(\mathrm{AUC}_{0-72 \mathrm{~h}}\right)$ of SNEDDS of

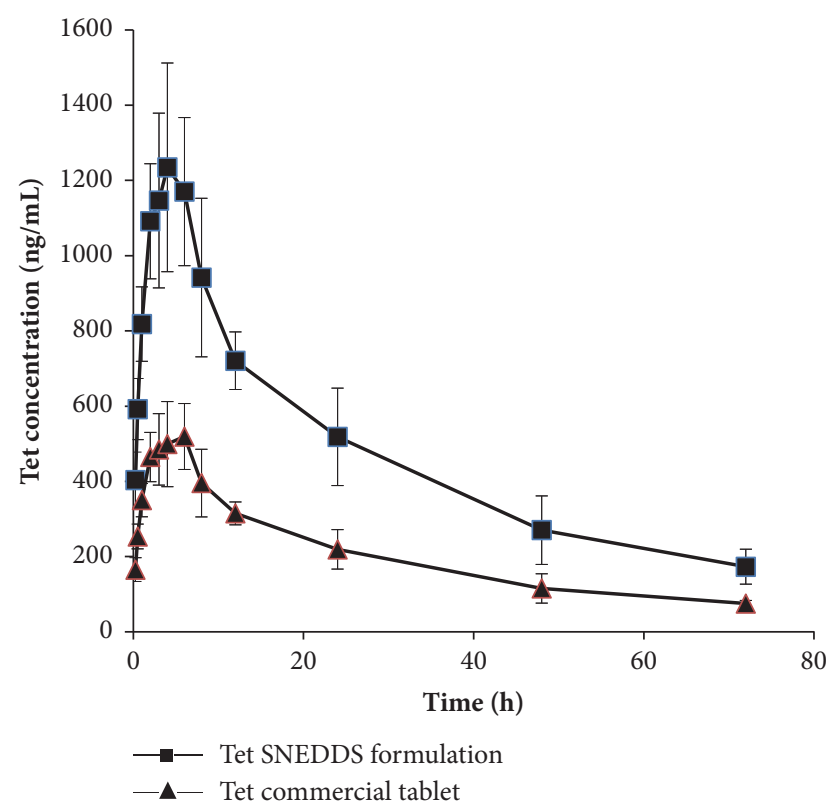

FIGURE 7: Plasma concentration profile of Tet after oral administration in male rats. Each value represents the mean $\pm S D(n=6$ and $10 \mathrm{mg} / \mathrm{kg}$ ).

Tet were higher than the Tet commercial tablet by $137.8 \%$ and $134.1 \%$, respectively. The relative bioavailability of the Tet SNEDDS formulation was 2.33-fold compared with the Tet commercial tablet. This indicated better systemic absorption of Tet from the SNEDDS formulation compared to the commercial tablet. The Tet SNEDDS formulation demonstrated almost similar mean residence time (MRT) (39.6 h) compared to the Tet commercial tablet MRT (39.9 h). Therefore, no prolonged action was found in the SNEDDS formulation. It was reasonable to conclude that SNEDDS enhances the absorption in vivo significantly.

\section{Discussion}

In our research, the potency of a self-microemusifying drug delivery system was successfully investigated in order to provide an effective system for delivery of Tet. Based on the results of the solubility tests in various excipients, we selected the optimal oil, cosolvent, surfactant, and cosurfactant. Through the construction of a ternary phase diagram, the optimal formulation of SNEDDS containing Tet was found to be the following: $40 \%(\mathrm{w} / \mathrm{w})$ oleic acid as oil, 15\% (w/w) SPC and 30\% (w/w) Cremophor RH-40 as surfactant, and $15 \%(\mathrm{w} / \mathrm{w})$ PEG400 as cosurfactant. The particle size, zeta-potential, stability, in vitro dissolution, and in vivo bioavailability of the resultant emulsion after self-emulsification were determined. The average droplet size of the optimal formulation was $19.75 \pm 0.37 \mathrm{~nm}$, and the average zeta-potential was $1.87 \pm 0.26 \mathrm{mv}$. With other new formulations of Tet such as nanoparticles (Tet-loaded PVP-b-PCL nanoparticles) [35], nanocapsules (tetrandrinephospholipid complex loaded lipid nanocapsules) [22], and microsphere (tetrandrine-loaded chitosan microsphere) [25], 
TABLE 3: Pharmacokinetic parameters of Tet SNEDDS formulation and Tet commercial tablet after single dose administration ( $\mathrm{n}=6$ ).

\begin{tabular}{|c|c|c|c|}
\hline Parameters & Unit & SNEDDS & Tablet \\
\hline $\mathrm{AUC}_{0-72 \mathrm{~h}}$ & $\mathrm{ng} / \mathrm{mL} \mathrm{h}$ & $33855.6 \pm 2024.3^{*}$ & $14459.4 \pm 3131.4$ \\
\hline $\mathrm{AUC}_{0-\infty}$ & $\mathrm{ng} / \mathrm{mL} \mathrm{h}$ & $41078.4 \pm 3621.2^{*}$ & $17604.0 \pm 4337.3$ \\
\hline $\mathrm{T}_{\max }$ & $\mathrm{h}$ & $3.8 \pm 1.2^{*}$ & $6.6 \pm 1.6$ \\
\hline $\mathrm{C}_{\max }$ & $\mathrm{ng} / \mathrm{mL}$ & $1234.8 \pm 39.7^{*}$ & $519.3 \pm 26.8$ \\
\hline MRT & $\mathrm{h}$ & $39.6 \pm 10.2$ & $39.9 \pm 12.1$ \\
\hline Relative bioavailability & & $233.3 \%$ & \\
\hline
\end{tabular}

${ }^{*} p<0.05$ compared to the Tet commercial tablet.

SNEDDS-Tet has a smaller particle size (size ranges of nanocapsules and microsphere: from $40 \mathrm{~nm}$ to $15 \mu \mathrm{m}$ ), equivalent solubility (solubility of nanocapsules in water: $8.28 \pm$ $0.37 \mu \mathrm{g} / \mathrm{mL}$ ), and a longer mean residence time (MRT of nanocapsules nanocapsules: $8.39 \pm 1.532 \mathrm{~h}$ ).

Encapsulation of drugs that are poorly soluble and have hydrophobic properties and poor distribution has been studied quite widely $[31,36]$. This study has some advantages over previous studies into the delivery of Tet because the SNEDDS-Tet can form a microemulsion spontaneously and the diameter of SNEDDS-Tet was smaller than those used previously [27], which might allow it to be more easily delivered into cells or to pass across some barriers. Recently, in order to overcome high cost of SNEDDS, a new, supersaturable self-emulsifying drug delivery system (S-SEDDS) was developed [37]. But some preparation methods of S-SEDDS cannot be used for Tet because of its thermal instability. Therefore, the focus of our research for Tet concentrates on SNEDDS.

Using mixed surfactants can achieve a rational value of hydrophile-lipophile balance (HLB) that using a single surfactant cannot achieve. The mixture of cremophor RH-40 and SPC can adjust the arrangement of the surfactant in the oil-water interface and improve the structure of the interfacial film, increase mobility and stability of the film, and so allow easier formation of a microemulsion [38]. In addition, selecting the appropriate surfactant HLB value can reduce the appearance of crystallization. The expected HLB of SNEDDS is usually within the range of 14-18, and the HLB of SNEDDS was 14-16 in this study. In vitro dissolution experiments revealed that the dissolution of Tet from SNEDDS was faster than the commercial tablet. The relative bioavailability of Tet SNEDDS was enhanced by $233 \%$. In addition to improving the solubility of the drug, there are a number of other biopharmaceutical properties that can affect the bioavailability of hydrophobic drugs. Surface active agents, dispersed particle size, lymphatic transport, lipolysis, and inhibition of intestinal metabolism can improve bioavailability. Thus, the system developed here could be used as an effective approach for enhancing the solubility and bioavailability of Tet and the Tet SNEDDS is worthy of further investigation.

\section{Conclusion}

In summary, self-nanoemulsifying drug delivery system composed of $40 \%$ (w/w) oleic acid as oil, 15\% (w/w) SPC and
$30 \%(w / w)$ Cremophor RH-40 as surfactant, and 15\% (w/w) $\mathrm{PEG}_{400}$ as cosurfactant was used to prepare the Tet SNEDDS. The results of in vitro dissolution study show that the dissolute rate of the prepared Tet SNEDDS in various dissolution media was remarkably faster than commercial tablet due to the small droplet size. And the results of pharmacokinetic study show that the prepared Tet SNEDDS achieved an approximately 2.33-fold increase in bioavailability compared with the commercial tablet. It could be concluded that selfnanoemulsifying drug delivery system of tetrandrine has the potential to improve the dissolution and oral bioavailability of poor water-soluble Tet.

\section{Data Availability}

The data used to support the findings of this study are available from the corresponding author upon request.

\section{Conflicts of Interest}

The authors declare that there are no conflicts of interest regarding the publication of this paper.

\section{Authors' Contributions}

Chunxia Liu and Li Lv contributed equally to this work.

\section{Acknowledgments}

This work was supported by National Natural Science Foundation of China (Grant no. 81503001); Guangdong Natural Science Foundation (Grant no. 2016A030313330); Guangdong province science and technology plan project public welfare fund and ability construction project (Grant nos. 2017A020215081, 2016A020215069, and 2016A020215063); Yixian Scientific Research Project Set Sail (Grant no. YXQH201706); Medical Scientific Research Foundation of Guangdong Province (Grant no. A2017062); the Science and Technology Foundation of Guangzhou (Grant no. 201707010103); Guangxi Provincial Department of science \& Technology (Grant no. 1346011-22); and Nanning Science \& Technology (Grant no. 20123117).

\section{References}

[1] Y.-T. Huang and C.-Y. Hong, “Tetrandrine," Cardiovascular Drug Reviews, vol. 16, no. 1, pp. 1-15, 1998. 
[2] K. Chen, A. Chen, R. Anderson, and C. Rose, "The pharmacological action of tetrandrine, an alkaloid of han-fang-chi," Chinese Journal Of Physiology, vol. 11, pp. 13-34, 1937.

[3] G. Wang, J. R. Lemos, and C. Iadecola, "Herbal alkaloid tetrandrine: From an ion channel blocker to inhibitor of tumor proliferation," Trends in Pharmacological Sciences, vol. 25, no. 3, pp. 120-123, 2004.

[4] N. Sekiya, H. Hikiami, K. Yokoyama et al., "Inhibitory effects of Stephania tetrandra S. Moore on free radical-induced lysis of rat red blood cells," Biological \& Pharmaceutical Bulletin, vol. 28, no. 4, pp. 667-670, 2005.

[5] L.-H. Fang, Y.-H. Zhang, and B.-S. Ku, "Fangchinoline inhibited the antinociceptive effect of morphine in mice," Phytomedicine, vol. 12, no. 3, pp. 183-188, 2005.

[6] L. Pang and J. R. S. Hoult, "Cytotoxicity to macrophages of tetrandrine, an antisilicosis alkaloid, accompanied by an overproduction of prostaglandins," Biochemical Pharmacology, vol. 53, no. 6, pp. 773-782, 1997.

[7] R.-L. Bian, "Antiallergic effect and its mechanism of tetrandrine," European Journal of Pharmacology, vol. 183, no. 2, p. 227, 1990.

[8] H. Wang, S. D. Luo, and H. S. C. Chin, "Research progress in pharmacological actions of tetrandrine," Chinese Pharmaceutical Association, no. 12, pp. 10-12, 2000.

[9] H. Takemura, K. Imoto, H. Ohshika, and C.-Y. K. Wan, "Tetrandrine as a calcium antagonist," Clinical and Experimental Pharmacology and Physiology, vol. 23, no. 8, pp. 751-753, 1996.

[10] G. Wang and J. Lemos, "Tetrandrine: a new ligand to block voltage-dependent $\mathrm{Ca} 2+$ and $\mathrm{Ca}(+)$-activated $\mathrm{K}+$ channels," Life Sciences, vol. 56, no. 5, pp. 295-306, 1994.

[11] P. L. Schiff, "Bisbenzylisoquinoline alkaloids," Journal of Natural Products, vol. 50, no. 4, pp. 529-599, 1987.

[12] Y. C. Shen, C. J. Chou, W. F. Chiou, and C. F. Chen, "Antiinflammatory effects of the partially purified extract of radix Stephaniae tetrandrae: comparative studies of its active principles tetrandrine and fangchinoline on human polymorphonuclear leukocyte functions," Molecular Pharmacology, vol. 60, no. 5, pp. 1083-1090, 2001.

[13] R. Man-Ren, "Effects of tetrandrine on cardiac and vascular remodeling," Acta Pharmacologica Sinica, vol. 23, no. 12, pp. 1075-1085, 2002.

[14] R. H. Reist, R. D. Dey, J. P. Durham, Y. Rojanasakul, and V. Castranova, "Inhibition of proliferative activity of pulmonary fibroblasts by tetrandrine," Toxicology and Applied Pharmacology, vol. 122, no. 1, pp. 70-76, 1993.

[15] N. Bhagya and K. R. Chandrashekar, "Tetrandrine-A molecule of wide bioactivity," Phytochemistry, vol. 125, pp. 5-13, 2016.

[16] T. Chen, B. Ji, and Y. Chen, "Tetrandrine triggers apoptosis and cell cycle arrest in human renal cell carcinoma cells," Journal of Natural Medicines, vol. 68, no. 1, pp. 46-52, 2014.

[17] Q. Chen, Y. Li, Y. Shao et al., “TGF- $\beta 1 / P T E N / P I 3 K$ signaling plays a critical role in the anti-proliferation effect of tetrandrine in human colon cancer cells," International Journal of Oncology, vol. 50, no. 3, pp. 1011-1021, 2017.

[18] K. Guo and J. Cang, "Pharmacodynamic evaluation of novel tetrandrine-loaded chitosan microspheres," Letters in Drug Design and Discovery, vol. 14, no. 6, pp. 686-689, 2017.

[19] W. Qiu, A.-L. Zhang, and Y. Tian, "Tetrandrine triggers an alternative autophagy in DU145 cells," Oncology Letters, vol. 13, no. 5, pp. 3734-3738, 2017.
[20] M. Yao, B. Yuan, X. Wang et al., "Synergistic cytotoxic effects of arsenite and tetrandrine in human breast cancer cell line MCF7," International Journal of Oncology, vol. 51, no. 2, pp. 587-598, 2017.

[21] L. Ye, S. Hu, H. Xu et al., “The effect of tetrandrine combined with cisplatin on proliferation and apoptosis of A549/DDP cells and A549 cells," Cancer Cell International, vol. 17, no. 1, 2017.

[22] Y.-Q. Zhao, L.-P. Wang, C. Ma, K. Zhao, Y. Liu, and N.-P. Feng, "Preparation and characterization of tetrandrine-phospholipid complex loaded lipid nanocapsules as potential oral carriers," International Journal of Nanomedicine, vol. 8, pp. 4169-4181, 2013.

[23] C. Shi, S. Ahmad Khan, K. Wang, and M. Schneider, "Improved delivery of the natural anticancer drug tetrandrine," International Journal of Pharmaceutics, vol. 479, no. 1, pp. 41-51, 2015.

[24] C. Fan, X. Li, Y. Zhou et al., "Enhanced topical delivery of tetrandrine by ethosomes for treatment of arthritis," BioMed Research International, vol. 2013, Article ID 161943, 13 pages, 2013.

[25] K. Guo and J. Cang, "A novel tetrandrine-loaded chitosan microsphere: Characterization and in vivo evaluation," Drug Design, Development and Therapy, vol. 10, pp. 1291-1298, 2016.

[26] W. Tan, Y. Li, M. Chen, and Y. Wang, "Berberine hydrochloride: anticancer activity and nanoparticulate delivery system," International Journal of Nanomedicine, vol. 6, pp. 1773-1777, 2011.

[27] X. Tian, J.-B. Zhu, J.-S. Wang, and T.-F. Li, "Preparation of tetrandrine lipid emulsion and its therapeutic effect on bleomycin-induced pulmonary fibrosis in rats," Journal of China Pharmaceutical University, vol. 36, no. 3, pp. 225-229, 2005.

[28] X. Cao, J. Luo, T. Gong, Z.-R. Zhang, X. Sun, and Y. Fu, "Coencapsulated doxorubicin and bromotetrandrine lipid nanoemulsions in reversing multidrug resistance in breast cancer in vitro and in vivo," Molecular Pharmaceutics, vol. 12, no. 1, pp. 274-286, 2015.

[29] S. Bandopadhyay, B. Singh, R. Kapil, R. Singh, and O. P. Katare, "Self-emulsifying drug delivery systems (SEDDS): formulation development, characterization, and applications," Critical Reviews in Therapeutic Drug Carrier Systems, vol. 26, no. 5, pp. 427-521, 2009.

[30] A. R. Patel and P. R. Vavia, "Preparation and in vivo evaluation of SMEDDS (self-microemulsifying drug delivery system) containing fenofibrate," The AAPS Journal, vol. 9, no. 3, pp. E344E352, 2007.

[31] C. J. H. Porter, C. W. Pouton, J. F. Cuine, and W. N. Charman, "Enhancing intestinal drug solubilisation using lipid-based delivery systems," Advanced Drug Delivery Reviews, vol. 60, no. 6, pp. 673-691, 2008.

[32] L. Zhang, L. Zhang, M. Zhang et al., "Self-emulsifying drug delivery system and the applications in herbal drugs," Drug Delivery, vol. 22, no. 4, pp. 475-486, 2015.

[33] A. Karthik, G. Subramanian, A. Ranjith Kumar, and N. Udupa, "Simultaneous estimation of paracetamol and domperidone in tablets by reverse phase HPLC method," Indian Journal of Pharmaceutical Sciences, vol. 69, no. 1, pp. 142-144, 2007.

[34] F. X. I. L. LI Zhonghong, C. A. I. I. Ing, Y. Zhihong, Y. I. Jian, and D. G. Q. I. Guifen, "Pharmacokinetics of fangch inoline and tetrandrine in rats," China Journal of Chinese Materia Medica, vol. 34, no. 23, pp. 3110-3113, 2009.

[35] H. Xu, Z. Hou, H. Zhang et al., "An efficient Trojan delivery of tetrandrine by poly(N-vinylpyrrolidone)-block-poly(epsiloncaprolactone) (PVP-b-PCL) nanoparticles shows enhanced 
apoptotic induction of lung cancer cells and inhibition of its migration and invasion," International Journal of Nanomedicine, vol. 9, no. 1, pp. 231-242, 2014.

[36] T. Liu, X. Liu, and W. Li, "Tetrandrine, a Chinese plant-derived alkaloid, is a potential candidate for cancer chemotherapy," Oncotarget, vol. 7, no. 26, pp. 40800-40815, 2016.

[37] D. H. Lee, D. W. Yeom, Y. S. Song et al., "Improved oral absorption of dutasteride via Soluplus ${ }^{\circledR}$-based supersaturable selfemulsifying drug delivery system (S-SEDDS)," International Journal of Pharmaceutics, vol. 478, no. 1, pp. 341-347, 2015.

[38] B. Bahloul, M. A. Lassoued, and S. Sfar, "A novel approach for the development and optimization of self emulsifying drug delivery system using HLB and response surface methodology: Application to fenofibrate encapsulation," International Journal of Pharmaceutics, vol. 466, no. 1-2, pp. 341-348, 2014. 

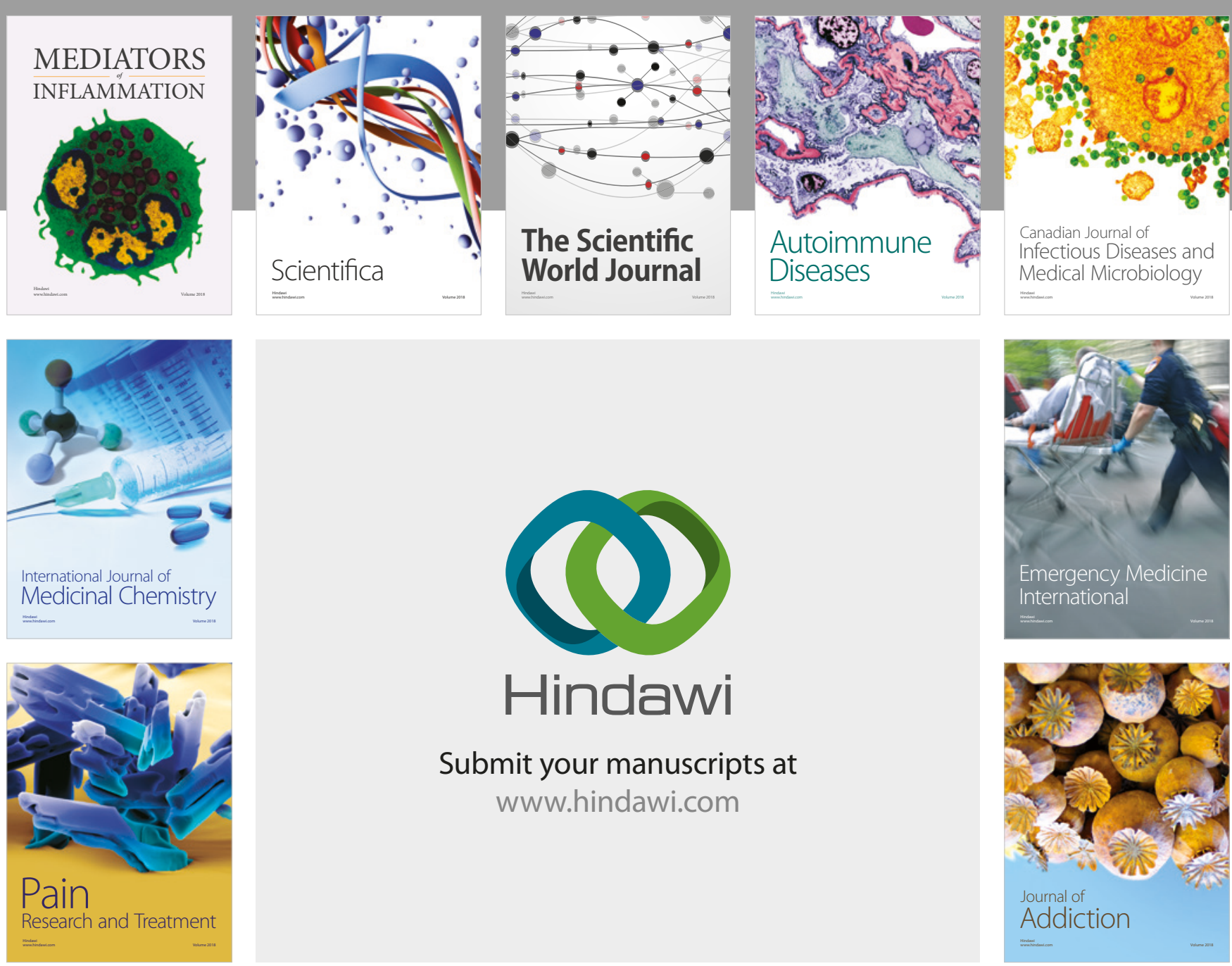

Canadian Journal of
Infectious Diseases and Medical Microbiology

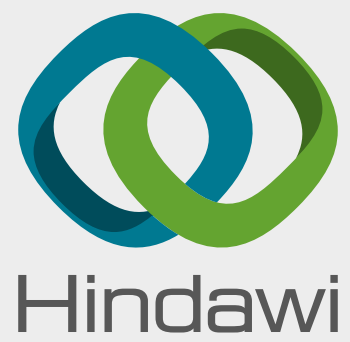

Submit your manuscripts at

www.hindawi.com
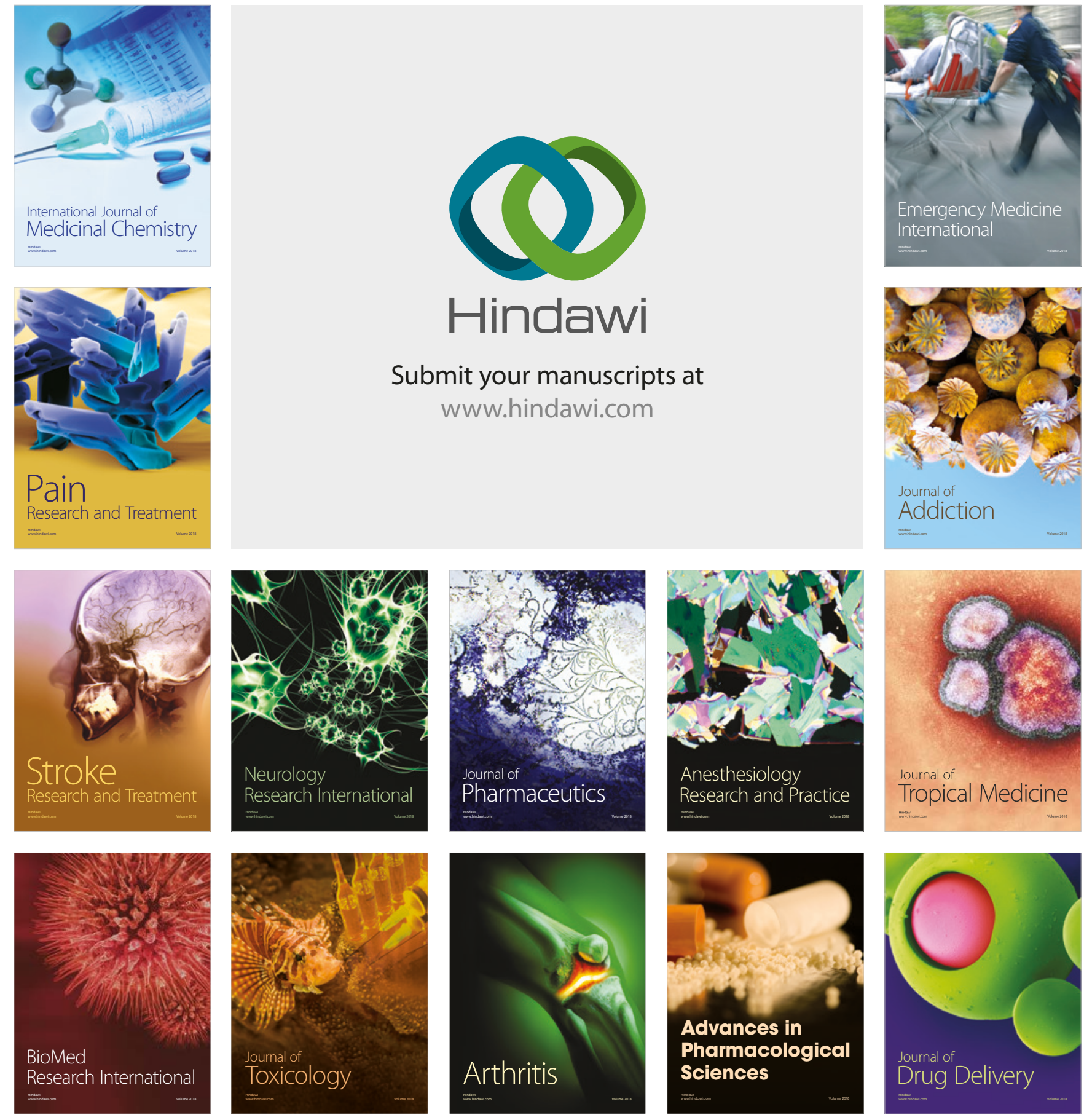